[Short Note]

\title{
QFP/quartz Adhesive Bonding with Surface Treatment for Physical Security of Edge Artificial Intelligence Devices
}

\author{
Hiro Nodaka*, Ami Tezuka*, Hiroyuki Kuwae*, Kosuke Yamada*, Morihisa Hoga**, Haruo Shimamoto**, \\ Shuichi Shoji*, and Jun Mizuno*** \\ *Waseda University, 3-4-1 Okubo, Shinjuku, Tokyo 169-8555, Japan \\ **National Institute of Advanced Industrial Science and Technology, 1-1-1 Umezono, Tsukuba 305-8560, Japan \\ ***Research Organization for Nano and Life Innovation, Waseda University, 513 Waseda tsurumakicho, Shinjuku, Tokyo 162-0041, Japan
}

(Received April 16, 2020; accepted June 12, 2020, published August 3, 2020)

\begin{abstract}
We studied a method of quad flat package (QFP)/quartz adhesive bonding. This bonding technology is required for developing physical security of edge artificial intelligence (AI) devices using a nanoartifact metrics (NAM) chip. Relationships between contact angle and bonding strength of QFP/adhesive or quartz/adhesive were investigated under several surface treatment methods including vacuum ultraviolet (VUV) treatments, and plasma treatments. VUV irradiation in the presence of nitrogen gas $\left(\mathrm{VUV} / \mathrm{N}_{2}\right)$ treatment reduced the contact angle of the QFP and quartz surface from $99.2^{\circ}$ to $5.0^{\circ}$, which improved QFP/adhesive bonding strength 5.7 times. Furthermore, the bonding strength of QFP/adhesive/quartz bonding with the VUV/ $\mathrm{N}_{2}$ treatment shows a shear strength of $14.2 \mathrm{MPa}$. It is 2.4 times higher value than that without the treatment. These results are a promising step for highly reliable $\mathrm{QFP} / \mathrm{quartz}$ bonding.
\end{abstract}

Keywords: Adhesive Bonding, VUV, Atmospheric Pressure Plasma, Edge AI Security

\section{Introduction}

Artificial intelligence (AI) is important for the information society where the amount of data exceeds human processing capacity. In recent years, concept of edge AI, which means AI algorithms are processed locally on each device, has attracted much attention. Security of the edge $\mathrm{AI}$ is one of the important factors to evolve AI merging society.[1] The edge AI device requires both software security and hardware security. Especially, the security for hardware has just begun to consider. There is a research about mounting of artifact metrics as a hardware security. [2] In this paper, we examined the mounting technology on the integrated circuit (IC) package, which is an AI edge device with using a nanoartifacts metrics (NAM) chip. The concept was shown in Fig. 1. The NAM chip physically provides high clone tolerance because of utilizing nanoscale pattern which is difficult to replicate. It is possible to add a security function in a post process by mounting the NAM chip in a bare state. However, the method of NAM chip mounting on the IC package with high reliability has not been studied yet.

In this study, we investigated a method of quad flat pack- age (QFP)/NAM chip adhesive bonding. A QFP is usually surface mounted on a printed wiring board with solder. Therefore, it is practical and has an advantage to add a security function with an exposed NAM chip after mounting of QFP. However, the bonding strength with sufficient durability of exposed QFP/NAM chip structure is an important factor. In a realistic edge AI device mounting structure, the size of the QFP molding area is $14 \mathrm{~mm}$ square, and of the pattern area of the nano-artifacts met-

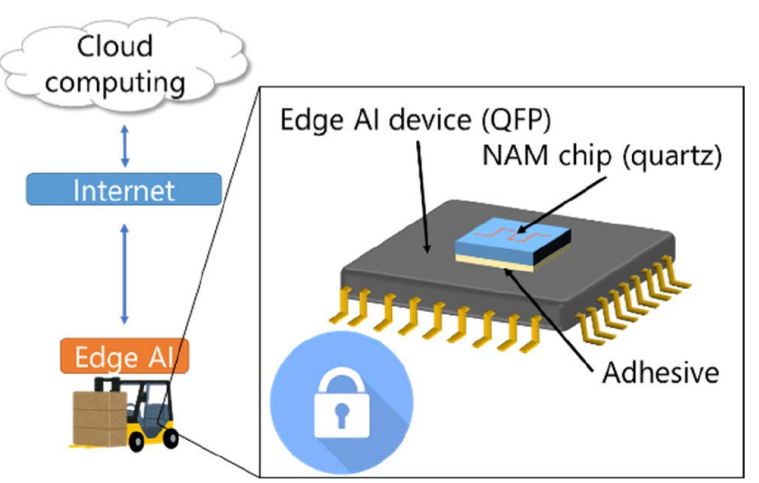

Fig. 1 Concept of physical security for AI edge devices using a NAM chip with an adhesive bonding method. 
rics is $20 \mu \mathrm{m}$ square.[3] In our experiment, we used the same size QFP and the bare quartz chip (5 mm square) instead of a NAM chip for simplicity. Aiming to improve the bonding strength, four types of surface treatments were performed before applying the adhesive: vacuum ultraviolet (VUV) irradiation in the presence of nitrogen gas $\left(\mathrm{VUV} / \mathrm{N}_{2}\right)$, VUV irradiation in the presence of oxygen gas $\left(\mathrm{VUV} / \mathrm{O}_{3}\right), \mathrm{Ar} / \mathrm{O}_{2}$ plasma, and Ar plasma. The hydrophilicity of the treated surfaces was evaluated by measuring the contact angle of water droplets. The bonding strength of QFP/adhesive and quartz/adhesive was examined by a shear test to clarify the effects of surface treatments on the each material. Further, QFP/adhesive/ quartz bonding was also performed to evaluate the bonding strength.

\section{Experimental Procedure}

\section{A. Surface treatment}

After organic cleaning with ultrasonication, four type of surface treatments were performed. The conditions of the surface treatments were summarized in Table 1 . The VUV treatment system (UER 20-172, Ushio, Inc.) consists of a lamp house and an irradiation chamber.[4] The VUV light directly reach and react with the sample surface in VUV/ $\mathrm{N}_{2}$ treatment. On the other hand, in $\mathrm{VUV} / \mathrm{O}_{3}$ treatment, the VUV light was absorbed by $\mathrm{O}_{2}$ to generate ozone and excited oxygen atoms, which react with the sample surface. Ar and $\mathrm{Ar} / \mathrm{O}_{2}$ plasma treatment was performed at atmospheric pressure (SHUTECH Co., Ltd.). All surface treatments were performed at a room temperature.

\section{B. Measurement of contact angle}

The hydrophilicity of the QFP and the quartz with and without surface treatments were measured by contact angle of distilled water drop (LCD-400S, Kyowa Interface

Table 1 Detailed condition of surface treatment.

\begin{tabular}{|c|c|c|}
\hline $\begin{array}{l}\text { Surface treatment } \\
\text { method }\end{array}$ & $\begin{array}{l}\text { Process } \\
\text { gas }\end{array}$ & Process power \\
\hline $\mathrm{VUV} / \mathrm{N}_{2}$ & $\begin{array}{c}\mathrm{N}_{2} \text { gas } \\
(20 \text { mbar })\end{array}$ & \multirow{2}{*}{$\begin{array}{l}\text { Power: } 10 \mathrm{~mW} / \mathrm{cm}^{2} \\
\quad \text { Time: } 10 \mathrm{~min} \\
\text { Wavelength: } 172 \mathrm{~nm}\end{array}$} \\
\hline $\mathrm{VUV} / \mathrm{O}_{3}$ & $\begin{array}{c}\mathrm{O}_{2} \text { gas } \\
(500 \text { mbar })\end{array}$ & \\
\hline $\begin{array}{c}\text { Ar plasma } \\
\text { (Atmospheric } \\
\text { pressure plasma) }\end{array}$ & Ar: 8 slpm & \multirow{2}{*}{$\begin{array}{c}\text { Power: } 200 \mathrm{~W} \\
\text { Speed: } 10 \mathrm{~mm} / \mathrm{s} \\
14 \text { times }\end{array}$} \\
\hline $\begin{array}{c}\text { Ar/O } \mathrm{O}_{2} \text { plasma } \\
\text { (Atmospheric } \\
\text { pressure plasma) }\end{array}$ & $\begin{array}{l}\text { Ar: } 8 \text { slpm } \\
\mathrm{O}_{2}: 2 \mathrm{sccm}\end{array}$ & \\
\hline
\end{tabular}

Science Co., Ltd.). The contact angles were measured within 5 minutes after the surface treatments.

C. Shear test for measuring bonding strength of $\mathrm{QFP} /$ adhesive, quartz/adhesive, QFP/adhesive/ quartz

We investigated the effects of surface treatments on the shear strength of QFP/adhesive, quartz/adhesive, and $\mathrm{QFP} /$ adhesive/quartz bonding. An adhesive of thermosetting resin was cured gradually at $120^{\circ} \mathrm{C}$ for $30 \mathrm{~min}, 150^{\circ} \mathrm{C}$ for $1 \mathrm{~h}$, and then $180^{\circ} \mathrm{C}$ for $4 \mathrm{~h}$. After the adhesive was completely cured, the bonding strength of QFP/adhesive, quartz/adhesive, QFP/adhesive/quartz were measured by a digital shear strength indicator (DPX-50T, IMADA).

\section{Results and Discussion}

Figure 2 shows the results of contact angle measurement. Initial contact angle of the QFP and the quartz surface were $99^{\circ}$ and $49^{\circ}$, respectively. The all treatment method reduced the contact angle of both QFP and quartz surfaces and realized hydrophilic surfaces. Especially, the QFP surface with the VUV/ $\mathrm{N}_{2}$ treatment showed the minimum contact angle of $5.0^{\circ}$. On the other hand, the all surface treatment method realized reduction of the contact angle less than $5^{\circ}$ for the quartz.

Figure 3 shows relationship between the contact angle and the bonding strength of QFP or quartz/adhesive bonding samples. The shear strength of QFP/adhesive bonding samples with all surface treatments increased in comparison to that without the treatment. The shear strength of QFP/adhesive bonding increased with decreasing the contact angle. Especially, VUV/ $\mathrm{N}_{2}$ treatment, which minimalized the contact angle of the QFP surface, maximized the shear strength of QFP/adhesive bonding 5.7 times. Although value of the bonding strengths of quartz/adhesive samples were not correspond with the value of the contact angle as previously

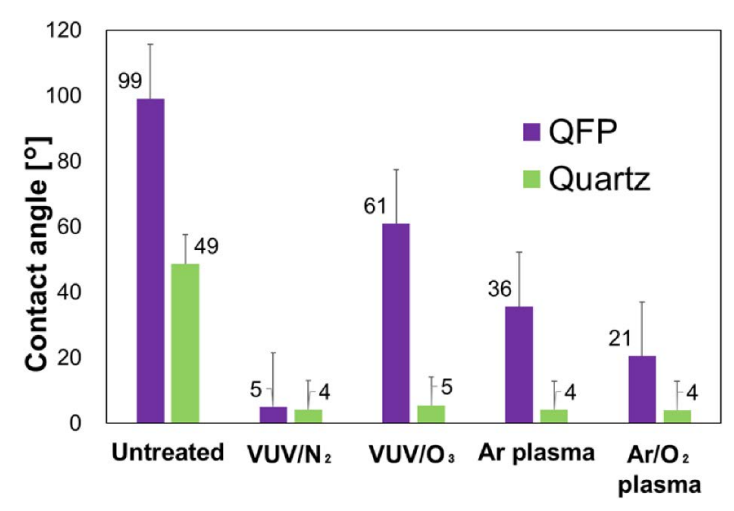

Fig. 2 Contact angle changes of QFP and quartz. 
reported.[5] QFP/adhesive/quartz bonding results with and without VUV/ $\mathrm{N}_{2}$ treatment were shown in Fig. 4. The bonding strength of $\mathrm{QFP} /$ adhesive/quartz bonding with

(a)

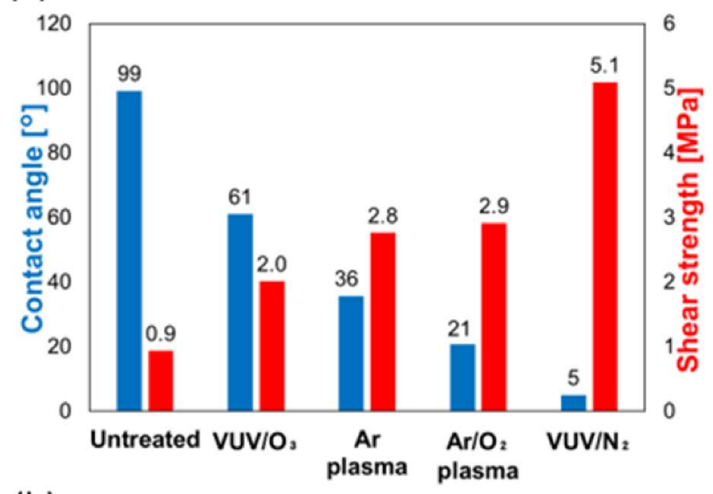

(b)

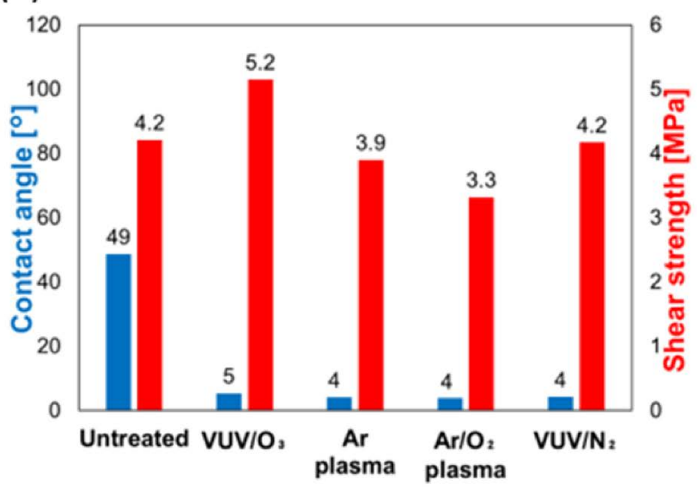

Fig. 3 Relationship between contact angle and shear strength of (a) QFP/adhesive and (b) quartz/adhesive bonding samples.

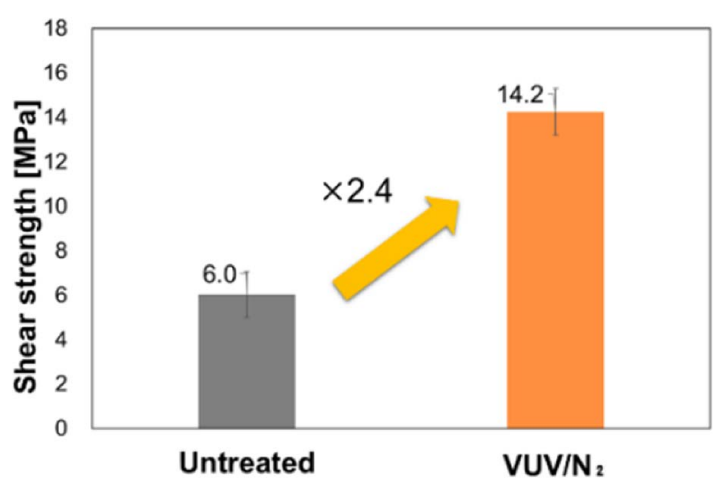

Fig. 4 Shear strength of QFP/adhesive/quartz bonding without and with $\mathrm{VUV} / \mathrm{N}_{2}$ treatment. the $\mathrm{VUV} / \mathrm{N}_{2}$ treatment shows a shear strength of 14.2 $\mathrm{MPa}$. It is 2.4 times higher value than that without the treatment.

\section{Conclusion}

In this study, we reported adhesive bonding method with surface treatment for a NAM chip mounting on an IC chip. The maximum bonding strength of QFP/adhesive was achieved with VUV/ $\mathrm{N}_{2}$ treatment which achieved the minimum contact angle. The proposed QFP/quartz bonding is promising for physical security of edge AI devices. In the future, we intend to investigate further the correlation between surface treatment and the bonding strength.

\section{Acknowledgment}

This paper is based on results obtained from a project, JPNP16007, commissioned by the New Energy and Industrial Technology Development Organization (NEDO). The authors thank for MEXT Nanotechnology Platform Support Project of Waseda University.

\section{References}

[1] J. Pan and Z. Yang, "Cybersecurity challenges and opportunities in the new "edge computing + IoT" world," Proceedings of the ACM International Workshop on Security in Software Defined Networks and Network Function Virtualization, pp. 29-32, 2018.

[2] T. Matsumoto and M. Aoyagi, "A Method of Applying Artifact-metrics for Enhancing Smart Card Security," IPSJ Journal, Vol. 46, pp. 2098-2106, 2005.

[3] T. Matsumoto, et al., "Optical nano artifact metrics using silicon random nanostructures,” Sci. Rep. Vol. 6, 32438, 2016.

[4] W. Fu, B. Ma, H. Kuwae, S. Shoji, and J. Mizuno, "Low-temperature poly(oxymethylene) direct bonding via self-assembled monolayer,” Jpn. J. Appl. Phys., Vol. 57, 2s1, 2018.

[5] M. M. R. Howlader, S. Suehara, and T. Suga, "Room temperature wafer level glass/glass bonding," Sens. Actuator A Phys., Vol. 127, pp. 31-36, 2006. 


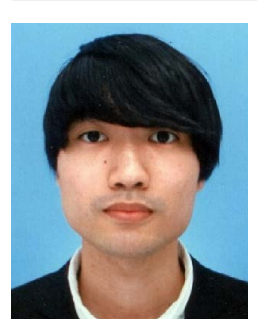

Hiro Nodaka was received the BE degree in the microsystems from Waseda University, Tokyo, Japan, in 2020. He is now a master course student of Waseda University. His current research interests is adhesive bonding.

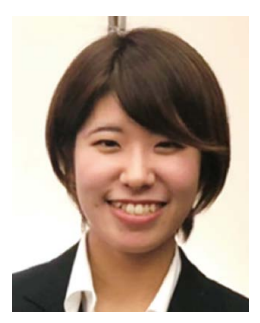

Ami Tezuka was received her BS degree in the field of microsystems from Waseda University in 2019. She is presently Master's course student at Waseda University. Her current interest is fabrication of surface acoustic wave device.

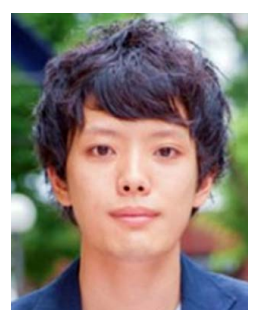

Hiroyuki Kuwae received his Ph.D. degree in the field of microsystems from Waseda University, Tokyo, Japan in 2017. He is currently an researcher at Research Organization for Nano and Life Innovation, Waseda University. His current research interests are MEMS/NEMS, flexible electronics, and low temperature bonding.

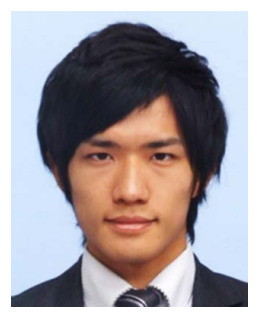

Kosuke Yamada received his BS and MS degree in the field of microsystems from Waseda University in 2018 and 2020, respectively. His research interest includes MEMS, and packaging technology.

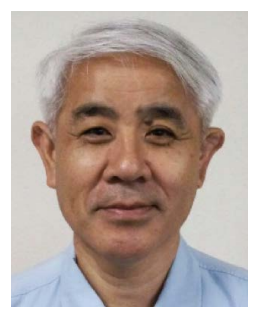

Morihisa Hoga received his B.S. degree in physics from Waseda University in 1974. And he received his $\mathrm{Ph} . \mathrm{D}$. degree in engineering from Kyusyu University in 2011. He worked at Hitachi Ltd. from1974 to 2000 in the field of photomask technology in semiconductor industry. He moved to Dai Nippon Printing Co., Ltd. and engaged in the field of nano fabrication from 2000 to 2016. He joined AIST in 2018. His current interest is in the application of nanofabrication technology to the field of security.

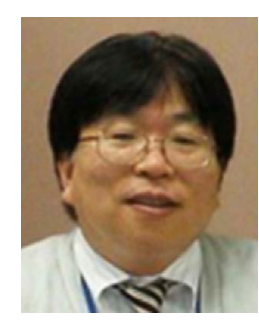

Haruo Shimamoto received the B.S. and M.S. degrees from Osaka University in 1980 and joined Semiconductor business unit in Mitsubishi Electric Corporation. The former business unit was merged to Renesas in 2003 , and he had been engaged about 28 years in development of semiconductor packaging and mass production. He joined National Institute of Advanced Industrial Science and Technology (AIST), Tsukuba, Japan, in 2014, where he has been engaged in the research of 3D integration packaging \& wafer bonding technologies. He is currently an invited researcher in the 3D integration system group, Device Technology Research Institute. He is a member of the Japan Institute of Electronics Packaging.

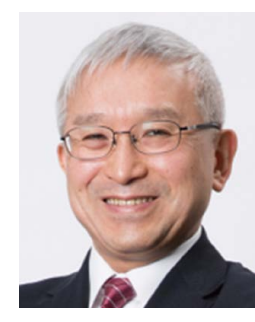

Shuichi Shoji received his Ph.D. degree in electronic engineering from Tohoku University in 1984 . He had been a research associate and associate professor at Tohoku University from 1984 to 1992. In 1994, he moved to Waseda University as an associate professor, and he is currently a professor of Department of Electronic and Photonic Systems and Department of Nanoscience and Nanoengineering, Waseda University. His current interests are micro-/nano-devices and systems for chemical/bio applications.

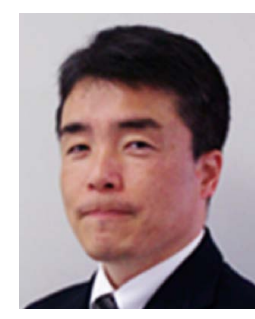

Jun Mizuno received his Ph.D. degree in applied physics from Tohoku University in 2000. He is currently a professor at Waseda University and works at Research Organization for Nano and Life Innovation where is a research institute of nano-science and engineering. His current interests are MEMSNEMS technology, low temperature bonding technology using plasma activation or excimer laser irradiation, printed electronics, and composite technology for UV or heat nanoimprint lithography combined with electrodeposition. 